\title{
The heteronuclear Efimov effect
}

\author{
K. Helfrich ${ }^{1, a}$ and H.-W. Hammer ${ }^{1}$ \\ Helmholtz-Institut für Strahlen- und Kernphysik (Theorie) \\ and Bethe Center for Theoretical Physics, Universität Bonn, 53115 Bonn, Germany
}

\begin{abstract}
Ultracold quantum gases with large scattering length show resonant enhancement of three-body loss rates when an Efimov trimer is at the scattering threshold. We calculate the three-body loss rates in heteronuclear mixtures of atoms for the case of large scattering length between the unlike atoms. Using zero-range interactions, we present results from the numerical solution of the integral equations for the recombination amplitude in momentum space and extract expressions for the recombination rate constants. Moreover, we calculate the relative positions of loss features for different sign of the scattering length and a first comparison with available experimental data is shown.
\end{abstract}

\section{Introduction}

The universal properties of particles with resonant shortrange interactions are a subject of intense research. Such systems are characterized by a large scattering length and display universal phenomena associated with a discrete scaling symmetry [1,2]. For identical bosons, Efimov discovered in 1970 that there are infinitely many trimer states with an accumulation point at the scattering threshold when the S-wave scattering length $a$ is tuned to the unitary limit $1 / a=0[3]:$

$$
E_{T}^{(n)}=\left(e^{-2 \pi / s_{0}}\right)^{n-n_{*}} \hbar^{2} \kappa_{*}^{2} / m,
$$

where $m$ is the mass of the particles, $s_{0} \approx 1.00624$, and $\kappa_{*}$ is the binding wavenumber of the branch of Efimov states labeled by $n_{*}$. The geometric spectrum in (1) is the signature of a discrete scaling symmetry with scaling factor $e^{\pi / s_{0}} \approx 22.7$. For a finite scattering length that is large compared to the range of the interaction, the universal properties persist but there will only be a finite number of Efimov states.

Ultracold atoms are an ideal tool to study such phenomena since the scattering length can be tuned experimentally using Feshbach resonances. Efimov trimers in ultracold atomic gases can be observed via their signature in three-body recombination rates $[4,5, ?, ?]$. Esry, Greene and Burke predicted a resonant enhancement of the threebody recombination rate at negative values of the scattering length [5]. The universal line shape for the resonance as a function of the scattering length was derived in [8]. Kraemer et al. provided the first evidence for Efimov trimers in an ultracold gas of ${ }^{133} \mathrm{Cs}$ atoms by observing the resonant enhancement of three-body recombination caused by the trimers [9]. In a subsequent experiment with a mixture of ${ }^{133} \mathrm{Cs}$ atoms and dimers, Knoop et al. observed a

\footnotetext{
a e-mail: helfrich@hiskp.uni-bonn.de
}

resonance in the loss of atoms and dimers [10] which can be explained by an Efimov trimer crossing the atom-dimer threshold [11]. The universal properties arising from the large scattering length are also manifest in four-body observables. Platter and Hammer discovered that there is a pair of universal tetramer states associated with every Efimov trimer and calculated their binding energies in the vicinity of the unitary limit [12]. Von Stecher, D'Incao and Greene mapped out their spectrum for all scattering lengths and pointed out that resonant enhancement of 4-body recombination would provide a signature for these tetramers [13]. This signature was subsequently observed in an ultracold gas of ${ }^{133} \mathrm{Cs}$ atoms by Ferlaino et al. [14].

Several recent experiments have also obtained evidence of Efimov physics with other bosonic atoms. Zaccanti et al. measured the three-body recombination rate and the atomdimer loss rate in an ultracold gas of ${ }^{39} \mathrm{~K}$ atoms [15]. They observed loss features at large positive and negative values of the scattering length with positions in agreement with the discrete scaling symmetry. Gross et al. measured the three-body recombination rate in an ultracold system of ${ }^{7} \mathrm{Li}$ atoms [16]. They observed a three-atom loss resonance and a three-body recombination minimum in the same universal region on different sides of a Feshbach resonance. Their positions are consistent with the universal predictions with discrete scaling factor 22.7. Using ultracold ${ }^{7} \mathrm{Li}$ atoms as well, Pollack et al. [17] observed eleven three- and fourbody loss features in the inelastic loss spectrum. Their relative locations on either side agree well with universal theory, while a systematic deviation from universality appears when comparing features across the resonance. The origin of this deviation is not understood.

The Efimov effect can also occur for fermionic atoms with at least three spin states. The first experimental studies of many-body systems of ${ }^{6} \mathrm{Li}$ atoms in the three lowest hyperfine states have recently been carried out by Ottenstein et al. [18] and by Huckans et al. [19]. Theoretical calcu- 
lations of the three-body recombination rate supported the interpretation that the narrow loss feature arises from an Efimov trimer crossing the three-atom threshold [20-23]. Very recently, another narrow loss feature was discovered in a much higher region of the magnetic field by Williams et al. [24] and by Jochim and coworkers [25]. In this region, the scattering length is much larger and several recombination features have been predicted using the universal theory [26].

In this paper, we focus on heteronuclear systems with two species of atoms where only the interspecies scattering length is large. In this case only two of the three pair scattering lengths are large and the preferred scaling factor is 1986.1 for equal mass particles. Such systems offer the opportunity to engineer the preferred scaling factor by adjusting the mass ratio of the atoms [27]. In the case of two heavy atoms and one light one, the scaling factor can become smaller than the value 22.7 for identical bosons. A first experiment measuring three-body losses in a heteronuclear mixture of ${ }^{41} \mathrm{~K}$ and ${ }^{87} \mathrm{Rb}$ atoms was carried out by Barontini et al. [28]. We will apply our theory to this system and address other combinations in planned experiments.

\section{Method}

In this section, we set up the effective field theory (EFT) method which will be used in the following. This EFT provides a convenient implementation of the universal theory for large scattering length. We will set $\hbar=1$ but restore the factors of $\hbar$ later on in our expressions for the recombination rate constants. We consider a system consisting of two types of bosonic atoms labelled species 1 and species 2. Assuming that the intraspecies interactions are not resonant ${ }^{1}$, we neglect these interactions. The only relevant interaction therefore takes place in between atoms of type 1 and 2 . Their reduced mass will be denoted by $\mu=m_{1} m_{2} /\left(m_{1}+m_{2}\right)$, where $m_{i}$ is the mass of atom species $i$. We also introduce an effective dimer field $d$ which couples to atoms of type 1 and 2. Furthermore, we will consider only trimers which consist of one atom of species 1 and two atoms of species 2 . The physics of trimers consisting of one atom of species 2 and two atoms of species 1 can be obtained by exchanging the labels 1 and 2 . We therefore include only the interactions between dimers and atoms of species 2. Hence, our effective Lagrangian reads:

$$
\begin{array}{r}
\mathcal{L}=\psi_{1}^{\dagger}\left(i \partial_{t}+\frac{\nabla^{2}}{2 m_{1}}\right) \psi_{1}+\psi_{2}^{\dagger}\left(i \partial_{t}+\frac{\nabla^{2}}{2 m_{2}}\right) \psi_{2} \\
+g_{2} d^{\dagger} d-g_{2}\left(d^{\dagger} \psi_{1} \psi_{2}+\psi_{1}^{\dagger} \psi_{2}^{\dagger} d\right)-\frac{g_{3}}{4} d^{\dagger} d \psi_{2}^{\dagger} \psi_{2}+\ldots,
\end{array}
$$

where the dots represent higher order derivative interactions. $g_{2}$ and $g_{3}$ are the bare two- and three-body coupling constants.

From this Lagrangian, we can deduce Feynman rules and obtain expressions for the full dimer propagator and

1 This requires the intraspecies scattering lengths to be comparable to the interspecies van-der-Waals length $\ell_{v d W}$ or smaller.

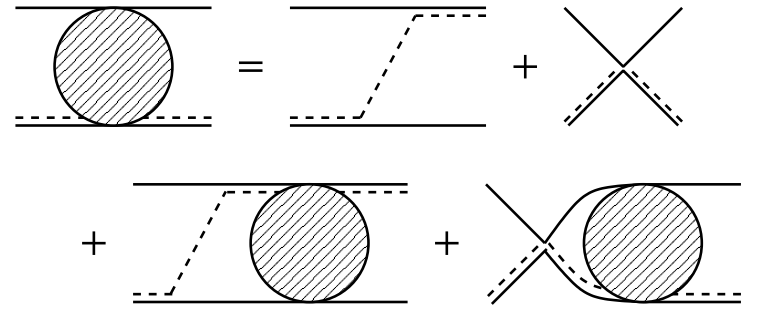

Fig. 1. Integral equation for the atom-dimer scattering amplitude $\mathcal{A}$. Solid (dashed) lines denote atom species 2 (1). Mixed double lines stand for the full dimer propagator.

the three-body integral equation. (See Ref. [1] for details on the derivation.) For the full dimer propagator, we get:

$$
D\left(P_{0}, \mathbf{P}\right)=\frac{2 \pi}{g_{2}^{2} \mu}\left[\frac{1}{a}-\sqrt{-2 \mu\left(P_{0}-\frac{P^{2}}{2 M}\right)-i \epsilon}\right]^{-1},
$$

where $P=|\mathbf{P}|$ and the total mass of the dimer equals $M=$ $m_{1}+m_{2}$. The dimer wave function renormalization is given by $Z_{D}^{-1}=g_{2}^{2} a \mu^{2} /(2 \pi)$.

The scattering between a dimer and an atom is described by the integral equation shown in Fig. 1. Using the Feynman rules derived from Eq. (2) and projecting on relative $\mathrm{S}$-waves, we have:

$$
\begin{aligned}
\mathcal{A}(p, k ; E)= & \frac{2 \pi m_{1}}{a \mu^{2}}\left[K(p, k)-\frac{g_{3}}{4 m_{1} g_{2}^{2}}\right] \\
& +\frac{m_{1}}{\pi \mu} \int_{0}^{\Lambda} d q q^{2}\left[K(p, q)-\frac{g_{3}}{4 m_{1} g_{2}^{2}}\right] \\
& \times \frac{\mathcal{A}(q, k ; E)}{-\frac{1}{a}+\sqrt{-2 \mu\left(E-\frac{q^{2}}{2 \mu_{A D}}\right)-i \epsilon}},
\end{aligned}
$$

where $\mu_{A D}=m_{2}\left(m_{1}+m_{2}\right) /\left(2 m_{2}+m_{1}\right)$ is the reduced mass of an atom and a dimer. The momenta $p(k)$ denote the relative momenta of the incoming (outgoing) atom-dimer pair and $E$ is the total energy. The contribution of the $\mathrm{S}$ wave projected one-atom exchange is given by

$$
K(p, q)=\frac{1}{2 p q} \ln \left[\frac{p^{2}+q^{2}+2 p q \frac{\mu}{m_{1}}-2 \mu E-i \epsilon}{p^{2}+q^{2}-2 p q \frac{\mu}{m_{1}}-2 \mu E-i \epsilon}\right],
$$

and the contribution of the three-body coupling $g_{3}$ can be written as

$$
\frac{g_{3}}{4 m_{1} g_{2}^{2}}=-\frac{H(\Lambda)}{\Lambda^{2}}
$$

where $H(\Lambda)$ is a dimensionless log-periodic function of the cutoff $\Lambda$ that depends on a three-body parameter $\Lambda_{*}$ [?]. The discrete scaling factor $\exp \left(\pi / s_{0}\right)$ depends on the ratio of masses of the particles, $\delta=m_{1} / m_{2}$, through the value of $s_{0}$. It is determined by the equation

$$
s_{0} \cosh \left(\pi s_{0} / 2\right)-2 \sinh \left(\phi s_{0}\right) / \sin (2 \phi)=0,
$$


with

$$
\phi=\arcsin [1 /(1+\delta)] .
$$

For particles of equal mass, the solution is $s_{0} \approx 0.4137$ leading to the preferred scaling factor $\exp \left(\pi / s_{0}\right) \approx 1986.1$. Because of the log-periodicity of $H(\Lambda)$ one can always find a value of the cutoff $\Lambda$ with $H=0$. In practice, one can therefore simply omit the three-body coupling in leading order calculations and use the cutoff $\Lambda$ as a three-body parameter [?]. We will use this strategy in the following. For fixed $\delta$, the values of $\Lambda$ and $\Lambda_{*}$ are related by a multiplicative constant.

The scattering amplitude $\mathcal{A}$ has simple poles at the three-body bound state energies $E=-B_{t}<0$. The energies can be obtained from the solution of a homogeneous integral equation for the bound state amplitude $\mathcal{B}$ :

$$
\mathcal{B}\left(p ; B_{t}\right)=\frac{m_{1}}{\pi \mu} \int_{0}^{\Lambda} \frac{d q q^{2} K(p, q) \mathcal{B}\left(q ; B_{t}\right)}{-\frac{1}{a}+\sqrt{2 \mu\left(B_{t}+\frac{q^{2}}{2 \mu_{A D}}\right)}},
$$

which only has non-trivial solutions for three-body binding energies $B_{t}>0$. In the following, we will use Eqs. $(4,9)$ to derive three-body properties for heteronuclear mixtures.

\section{Numerical Results}

Few-body loss phenomena offer a unique window on scattering processes in ultracold quantum gases. In particular, few-body loss rates can provide evidence for few-body resonances through their enhancement of the loss process. The universal theory provides predictions for the relative positions of these loss resonances as a function of the scattering length. When more than one resonance is detected in the same universal region, these predictions can be tested in experiment with ultracold atoms. In this work, we focus on predictions for three-body loss resonances in heteronuclear mixtures.

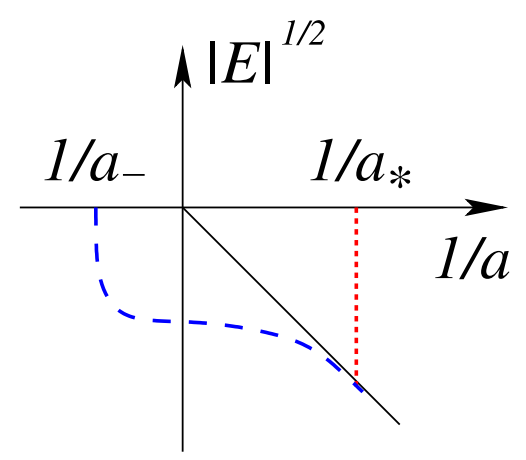

Fig. 2. Dependence of the trimer energy on the inverse scattering length $1 / a$ (dashed line). The parameters $a_{-}$and $a_{*}$ specify where the trimer state hits the three-atom and atom-dimer thresholds, respectively.

Alkali atoms typically form many deeply-bound dimer states with binding energies of order $\hbar^{2} /\left(2 \mu \ell_{v d W}^{2}\right)$. Their properties are insensitive to variations of the scattering length $a$ in the vicinity of the Feshbach resonances. If $a>0$, there is also a shallow dimer with binding energy $B_{d}=\hbar^{2} /\left(2 \mu a^{2}\right)$. The dynamics of the shallow dimer can be described by the universal theory while the inclusive effects of the deeply bound dimers on recombination can be accounted for by a complex three-body parameter as dicussed below. The scattering length dependence of a generic trimer energy is illustrated in Fig. 2. For negative scattering length the Efimov trimer hits the three-body scattering threshold of $E=0$ at $a=a_{-}$and leads to a resonance in the three-body recombination rate. This is due to enhanced scattering of three atoms via an Efimov resonance into a deeply bound dimer and a residual atom. The released binding energy transforms into kinetic energy of the atom and dimer which hence leave the trap. On the other side of the zero in the inverse scattering length, the trimer crosses the threshold for scattering of an atom and a shallow dimer. For energies above that threshold, atomdimer scattering is possible. Hence, at $a=a_{*}$, a resonance in atom-dimer scattering is expected. It was argued in [15, 28] that this could also be visible in three-atom scattering due to rescattering processes. Those would expell not only the three atoms involved in the original atom-dimer scattering process but many more leading to a measurable resonance. We will come back to this issue in Section 4.

The ratio of the two resonance positions $a_{*} /\left|a_{-}\right|$does not depend on the three-body parameter and is predicted from two-body physics alone. Solving the bound state equation (9) for $B_{t}=0, a<0$ and for $B_{t}=B_{d}, a>0$ with the same (arbitrary) cutoff $\Lambda$, one can extract the ratio $a_{*} /\left|a_{-}\right|$ as a function of the mass ratio, $\delta=m_{1} / m_{2}$. The results are shown in Fig. 3, where the solid curve shows the ratio if one trimer state is followed from $a<0$ to $a>0$ and the dashed line shows the ratio for two neighbouring trimers. The ratio of the two curves in Fig. 3 therefore reflects the behavior of the scaling factor $\exp \left(\pi / s_{0}\right)$ with $s_{0}$ given by Eq. (7) as a function of the mass ratio $\delta$ (see, e.g., Ref. [1] and references therein). For $\delta \gtrsim 1$ the scaling factor quickly gets very large. Therefore, a system with a small mass ratio is more likely to display a sequence of Efimov resonances.

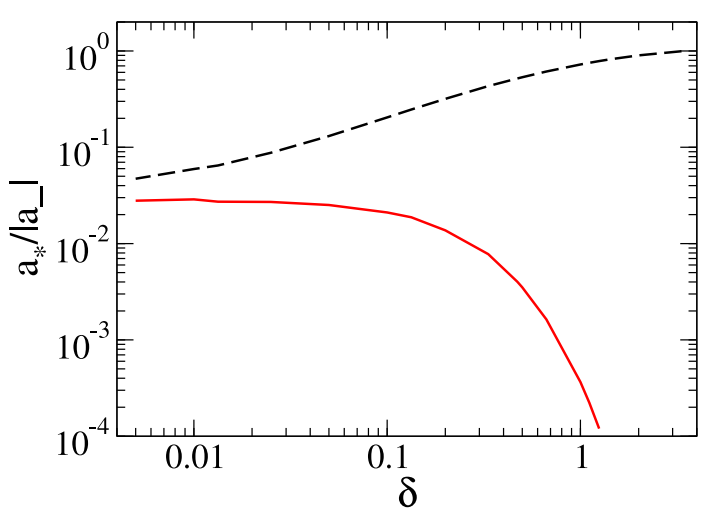

Fig. 3. Threshold ratio $a_{*} /\left|a_{-}\right|$in dependence of the mass ratio $\delta=m_{1} / m_{2}$ for following one trimer energy curve (solid line) or for two neighbouring trimers (dashed line). 
Next, we consider the universal predictions for the threebody loss rates in a heteronuclear system. We first turn to the case of positive scattering length, $a>0$, where the atoms can recombine into the shallow dimer and into deep dimers. The recombination into the shallow dimer can be related to the T-matrix element shown in Fig. 4(a). The (a)

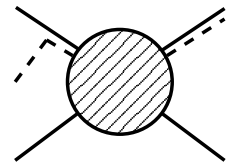

(b)

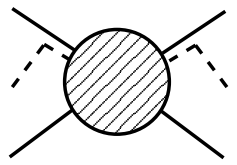

Fig. 4. Diagrammatic representation of the amplitude for (a) two atoms of type 2 and an atom of type 1 going to an atom of type 2 plus a dimer and (b) two atoms of type 2 and an atom of type 1 scattering elastically. Lines are as in Fig. 1.

event rate for inelastic scattering $\alpha$ is defined via

$$
\frac{d}{d t} n_{2}=2 \frac{d}{d t} n_{1}=-2 \alpha n_{1} n_{2}^{2}
$$

where $n_{i}$ denotes the atom number densities for the corresponding species.

The rate constant $\alpha_{s}$ for recombination into the shallow dimer is then given by

$$
\alpha_{s}=4 \mu_{A D} \sqrt{\frac{\mu_{A D}}{\mu}} a^{2}\left|\mathcal{A}\left(0, k_{D} ; 0\right)\right|^{2},
$$

with the dimer breakup momentum $k_{D}=\sqrt{\frac{\mu_{A D}}{\mu}} \frac{1}{a}$. If deep dimers are present, their effect on the recombination into the shallow dimer can be incorporated by analytically continuing the three-body parameter into the complex plane [20]. We thus make the substitution

$$
\Lambda \rightarrow \Lambda \exp \left(i \eta_{*} / s_{0}\right)
$$

in Eq. (4) where $\eta_{*}$ accounts for the effect of the deep dimers. A non-zero value of $\eta_{*}$ also generates the width of the Efimov trimers. By evaluating Eq. (11) numerically, we find that the known analytical formula for the three boson case [26] simply acquires a new mass-dependent overall coefficient. The modified analytical formula is hence

$$
\alpha_{s}=C(\delta) \frac{D\left(\sin ^{2}\left[s_{0} \ln \left(a / a_{* 0}\right)\right]+\sinh ^{2} \eta_{*}\right)}{\sinh ^{2}\left(\pi s_{0}+\eta_{*}\right)+\cos ^{2}\left[s_{0} \ln \left(a / a_{0 *}\right)\right]} \frac{\hbar a^{4}}{m_{1}}
$$

where $D=128 \pi^{2}(4 \pi-3 \sqrt{3})$ and the mass-dependent coefficient is denoted by $C(\delta)$. The parameter $a_{0 *}$ gives the position of the minimum in three-body recombination. The coefficient $C(\delta)$ is shown in Fig. 5. The error in the extraction of $C(\delta)$ from fitting Eq. (13) to our numerical results for $\alpha_{s}$ is of the order $10^{-3}$ for $\delta \leq 2$. For large values of $\delta$ the numerical extraction of $C$ becomes difficult since the scaling factor becomes very large. An analytic expression for $C(\delta)$ will be derived in [29]. The same coefficient $C$

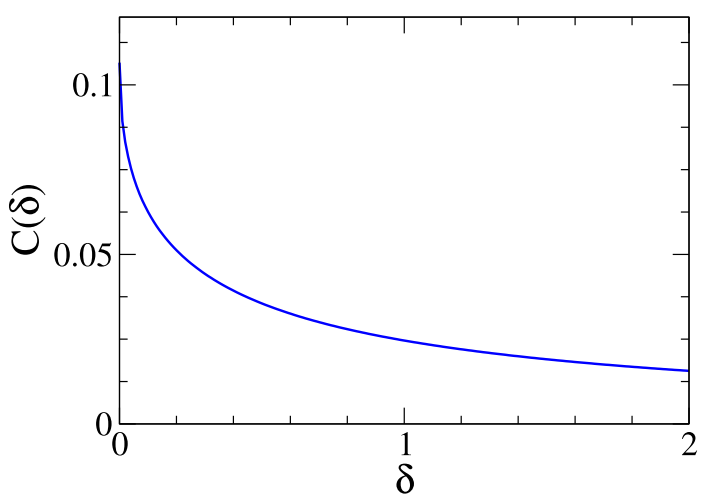

Fig. 5. The coefficient $C$ for the different three-body recombination rates in dependence of the mass ratio $\delta=m_{1} / m_{2}$.

will be shown to modify the recombination rates into deep dimers.

Although all calculations were conducted by varying the three-body parameter $\Lambda$, we give the results in dependence of the experimentally more relevant parameters $a_{* 0}$ and $a_{-}$. These parameters are reciprocally proportional to $\Lambda$ with a proportionality factor that depends on the mass ratio $\delta$. The parameter $a_{* 0}$ gives the position of the zero for recombination into shallow dimers if deep dimers are absent while $a_{-}$gives the position of the resonance for recombination into deep dimers at negative scattering length. Hence, they are easily accessible experimentally. For the case of three identical bosons, the connection between $a_{0 *}$ and $a_{*}$ is given by $a_{0 *, 3 B} \approx 4.5 a_{*, 3 B}$ [30]. This value yields a atom-dimer resonance quite close to the maximum of the three-body recombination. For different mass ratios this relation changes but we do not explicitly calculate it here. In [29], a general result for the relation between $a_{0 *}$ and $a_{-}$ is obtained. Together with the results for $a_{*} /\left|a_{-}\right|$in Fig. 3 this can be used to calculate the relation between $a_{0 *}$ on $a_{*}$ for various mass ratios.

The inclusive recombination rate for recombination into all dimers for $a>0$ can be obtained from the optical theorem. It relates the imaginary part of the forward T-matrix element (shown in Fig. 4(b)) for vanishing momenta to the event rate for inelastic scattering $\alpha$. This leads to the full recombination rate $\alpha_{f u l l}=\alpha_{s}+\alpha_{d}$ in the presence of deep dimers:

$$
\alpha_{\text {full }}=\operatorname{Im} T_{122 \rightarrow 122}=8 \pi a^{3} \operatorname{Im} \overline{\mathcal{A}}(0,0 ; 0),
$$

where $\overline{\mathcal{A}}$ denotes an appropriately infrared subtracted amplitude as in [26],

$$
\begin{aligned}
& \overline{\mathcal{A}}(p, k ; E)=\mathcal{A}(p, k ; E)-\frac{4 \pi(1+\delta)}{m_{1} a p^{2}} \\
& +\frac{4 \pi(1+\delta)^{2}}{m_{1} p} \arcsin [1 /(1+\delta)] \\
& +\frac{8 a}{m_{1}}\left[(1+\delta)^{2} \arcsin [1 /(1+\delta)]-\sqrt{\delta(2+\delta)}\right] \ln p .
\end{aligned}
$$


By comparison with Eq. (13), we find that the rate constant for the recombination into deep dimers is

$$
\alpha_{d}=C(\delta) \frac{D \operatorname{coth}\left(\pi s_{0}\right) \cosh \left(\eta_{*}\right) \sinh \left(\eta_{*}\right)}{\sinh ^{2}\left(\pi s_{0}+\eta_{*}\right)+\cos ^{2}\left[s_{0} \ln \left(a / a_{0 *}\right)\right]} \frac{\hbar a^{4}}{m_{1}}
$$

with the same coefficients $C(\delta)$ and $D$ as in Eq. (13). Apart from the overall $a^{4}$ coefficient, this recombination rate is found to be nearly independent of $a$.

In the case of three identical bosons, simplified expressions for the analog of Eqs. (13) and (16) exist (see, e.g., Ref. [1]). These expressions are only valid if $\exp \left(2 \pi s_{0}\right) \gg$ 1 , which is not the case throughout the whole range of mass ratios. Similar approximate expressions could be derived in our case.

For $a>0$ it is also possible to prepare a mixture of atoms and dimers, see e.g. Ref. [10]. The scattering length for atom-dimer scattering can be calculated from the solution of Eq. (4) via

$$
a_{A D}=-\frac{\mu_{A D}}{2 \pi} \mathcal{A}\left(0,0 ;-\frac{1}{2 \mu a^{2}}\right) .
$$

In analogy to the three-boson case, we can parametrize the exact numerical results using

$$
a_{A D}=\left(C_{1}(\delta)+C_{2}(\delta) \cot \left[s_{0} \ln \left(a / a_{*}\right)\right]\right) a .
$$

where the two coefficients $C_{1}(\delta)$ and $C_{2}(\delta)$ are shown in Fig. 6. Again, the error in the extraction of $C_{1}(\delta)$ and $C_{2}(\delta)$ from the exact numerical solution is of the order of $10^{-3}$. The atom-dimer scattering length displays a resonance at

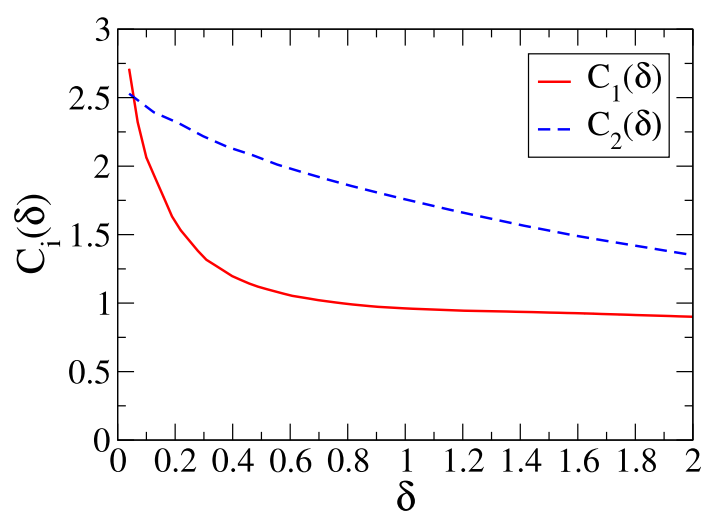

Fig. 6. The coefficients $C_{1}(\delta)$ and $C_{2}(\delta)$ in the expression (18) for the mass dependence of $a_{A D}$ as a function of the mass ratio $\delta=m_{1} / m_{2}$.

$a=a_{*}$ where an Efimov trimer hits the atom-dimer threshold. The effect of deep dimers on the elastic scattering process can again be incorporated by the parameter $\eta_{*}$. This parameter generates an imaginary part of the scattering length and is most conveniently introduced by replacing $a_{*} \rightarrow a_{*} \exp \left(-i \eta_{*} / s_{0}\right)$ analog to Eq. (12). The relaxation rate $\beta$ defined via

$$
\frac{d}{d t} n_{A}=\frac{d}{d t} n_{D}=-\beta n_{A} n_{D}
$$

is then given by [8]

$$
\beta=-\frac{4 \pi \hbar}{\mu_{A D}} \operatorname{Im} a_{A D} .
$$

Efremov and collaborators previously derived Eq. (18) for the atom-dimer scattering length using a BornOppenheimer approximation for two heavy atoms and a light one [31]. For $\delta=0.080$ corresponding to the ${ }^{7} \mathrm{Li}^{-}{ }^{87} \mathrm{Rb}$ system, our values for the coefficients $C_{1}$ and $C_{2}$ agree with the values given in [31] to within $2-3 \%$ (see Table 1 ). However, we find $s_{0}=1.523$ compared to $s_{0}=1.322$ in [31].

We now turn to negative scattering length $a<0$. In this case, there is no shallow dimer and only recombination into deep dimers can occur. We make again use of the optical theorem which now reads

$$
\alpha_{d}=\operatorname{Im} T_{122 \rightarrow 122}=8 \pi a^{3} \operatorname{Im} \overline{\mathcal{A}}(0,0 ; 0) .
$$

The three-atom recombination rate into deep dimers for $a<0$ is

$$
\alpha_{d}=C(\delta) \frac{4677.4 \sinh \left(2 \eta_{*}\right)}{\sin ^{2}\left[s_{0} \ln \left(a / a_{-}\right)\right]+\sinh ^{2}\left(\eta_{*}\right)} \frac{\hbar a^{4}}{m_{1}},
$$

with $C(\delta)$ as in Eq. (13). The numerical factor is taken from [20]. Note that Eq. (22) is only valid for $\delta \leq 0.5$. The exact prefactor for all values of $\delta$ will be derived in [29].

The coefficient $C(\delta)$ is largest for small mass ratios and quickly drops off for larger ratios. Hence, not only is the scaling factor more favorable for seeing multiple Efimov resonances for small mass ratios but also the three-body recombination rate for negative scattering length shows a more pronounced effect for small $\delta$.

\section{Comparison to experiment}

We now apply our results to experiments with ultracold atoms. In the group of Inguscio and Minardi in Florence, a mixture of ${ }^{87} \mathrm{Rb}$ and ${ }^{41} \mathrm{~K}$ was investigated [28]. By scanning through the scattering length and monitoring the number of atoms of both species, three loss resonances were observed. At negative scattering length, they identified a $\mathrm{K}-\mathrm{Rb}-\mathrm{Rb}$ resonance at $a=-246 a_{0}$ and a K-K-Rb resonance at $a=-22000 a_{0}$, where $a_{0}$ is the Bohr radius. Moreover, the third resonance at $a=667 a_{0}$ in the K-Rb$\mathrm{Rb}$ channel was attributed to enhanced atom-dimer scattering. This process was assumed to contribute to threebody losses through multiple rescattering processes. The same mechanism was also assumed in [15]. An independent confirmation of this resonance in a system prepared directly out of $\mathrm{K}-\mathrm{Rb}$ dimers and $\mathrm{Rb}$ atoms would be useful, however. Unfortunately, the short lifetime of the heteronuclear dimers makes such a confirmation difficult [32]. The interspecies van-der-Waals length in the $\mathrm{K}-\mathrm{Rb}$ system is $\ell_{v d W}=72 a_{0}$ such that these resonances should be within the range of validity of the universal theory.

Assuming the observed $\mathrm{K}-\mathrm{Rb}-\mathrm{Rb}$ features are indeed due to Efimov resonances, one can extract the ratio $a_{*} /\left|a_{-}\right|=$ 


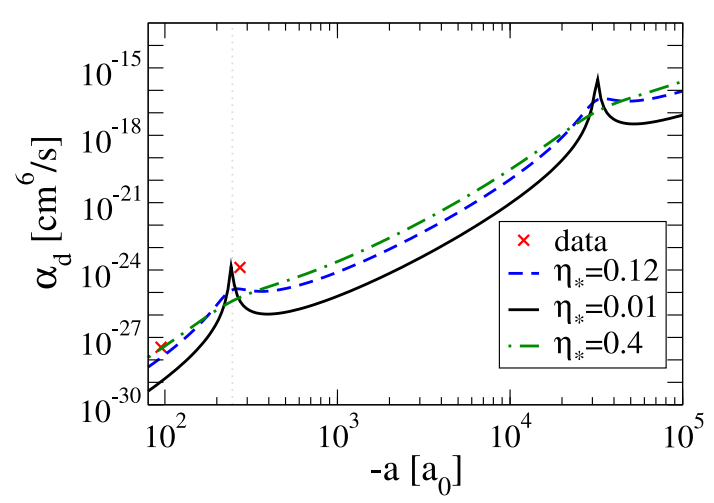

Fig. 7. $\alpha_{d}$ for the resonance at $a=-246 a_{0}$ (indicated by the dotted light grey line). The (blue) dashed curve is calculated for $\eta_{*}=0.12$ that was found in [28] whereas the (black) solid line is for $\eta_{*}=0.01$ and the (green) dash-dotted line is for $\eta_{*}=0.04$. Data points are taken from $[28,32]$, see text.

2.7 from this experiment. The theoretical prediction obtained in Sec. 3 is $a_{*} /\left|a_{-}\right|=0.52$. In view of the large scaling factor $\exp \left(\pi / s_{0}\right)=131$, the discrepancy is not too large but no definite conclusion can be drawn at the moment. Note also that relatively small discrepancies in the absolute values of $a_{*}$ and $a_{-}$can lead to substantial differences in the ratio of the two.

Apart from this ratio, the universal theory also predicts the size of the recombination rates. If the position and width of one resonance are known, all others in the universal region can be predicted. Unfortunately, the three-body recombination rate was measured in $[28,33]$ for only two values of $a$, such that a comparison with our calculation also is rather inconclusive. Fig. 7 shows $\alpha_{d}$ for the K-Rb$\mathrm{Rb}$ resonance at $a=-246 a_{0}$ where only the recombination into deep dimers can occur. With the mass ratio $\delta=$ 0.471 , we find for the coefficient $C(0.471)=0.037$. The (blue) dashed curve is calculated using the value $\eta_{*}=0.12$ extracted in [28]. The data point close to the resonance is taken from $[28,33]$, whereas the one further away from resonance gives an upper limit of the recombination rate [32]. In order to illustrate the sensitivity to $\eta_{*}$, we show the results for two alternative values: $\eta_{*}=0.01$ ((black) solid line) and $\eta_{*}=0.4$ ((green) dash-dotted line). The discrepancy between the measured recombination rate at the resonance and our result for $\eta_{*}=0.12$ is about one order of magnitude. In order to resolve this discrepancy, more measurements around the resonance position are required. Such data would allow for a more precise determination of the width parameter $\eta_{*}$ and a test of the resonance shape predicted by the universal theory. All relevant parameters for the ${ }^{41} \mathrm{~K}-{ }^{87} \mathrm{Rb}$ system are summarized in Table 1 . In the table, we always assume the single atom in a trimer to be from the lighter species since the opposite case has a less favorable scaling factor.

The Tübingen group of Zimmermann has carried out experiments using ${ }^{7} \mathrm{Li}^{87}{ }^{87} \mathrm{Rb}$ and ${ }^{6} \mathrm{Li}^{-}{ }^{87} \mathrm{Rb}$ mixtures. They already measured interspecies Feshbach resonances in the ${ }^{7} \mathrm{Li}^{-}{ }^{87} \mathrm{Rb}$ and in the ${ }^{6} \mathrm{Li}^{-}{ }^{87} \mathrm{Rb}$ systems $[34,35]$ and have reached quantum degeneracy [36]. With respect to the Efi-

\begin{tabular}{|l|l|l|l|l|}
\hline & ${ }^{7} \mathrm{Li}^{133}{ }^{13}$ & ${ }^{6} \mathrm{Li}^{87} \mathrm{Rb}$ & ${ }^{7} \mathrm{Li}^{-87} \mathrm{Rb}$ & ${ }^{41} \mathrm{~K}^{87} \mathrm{Rb}$ \\
\hline$\delta$ & 0.053 & 0.069 & 0.080 & 0.471 \\
$s_{0}$ & 1.850 & 1.635 & 1.523 & 0.6444 \\
$\exp \left(\pi / s_{0}\right)$ & 5.465 & 6.835 & 7.864 & 131.0 \\
$a_{*} /\left|a_{-}\right|$ & 0.13 & 0.16 & 0.18 & 0.52 \\
$C(\delta)$ & 0.072 & 0.068 & 0.066 & 0.037 \\
$C_{1}(\delta)$ & 2.54 & 2.33 & 2.22 & 1.13 \\
$C_{2}(\delta)$ & 2.52 & 2.5 & 2.47 & 2.08 \\
\hline
\end{tabular}

Table 1. Parameters for various heteronuclear mixtures. The single atom in the trimer is assumed to be from the lighter species.

mov effect, this experiment combines two interesting features. First, both mass ratios are quite small which implies a small scaling factor and hence is favorable to observing a sequence of Efimov resonances. Second, the possibility to switch between fermionic and bosonic lithium can be used to measure the heteronuclear Efimov effect in both systems and compare the results. A mixture of ${ }^{7} \mathrm{Li}$ and ${ }^{133} \mathrm{Cs}$ atoms was created in Heidelberg [37]. This system has a very small scaling factor and is another good candidate for observing Efimov physics in heteronuclear mixtures. Our results for the Efimov parameters of these systems are summarized in Table 1.

In Fig. 8, we show our prediction for $\alpha_{d}$ in the ${ }^{7} \mathrm{Li}^{87} \mathrm{Rb}$ system in units of $\hbar a^{4} / m_{L i}$ for negative interspecies scattering length $a$ for different values of $\eta_{*}$. The (red) dashed curve gives our result for $\eta_{*}=0.05$ while the (blue) solid curve gives the result for $\eta_{*}=0.5$. The resonance position $a_{-}$cannot be predicted by the universal theory and has to be taken from experiment.

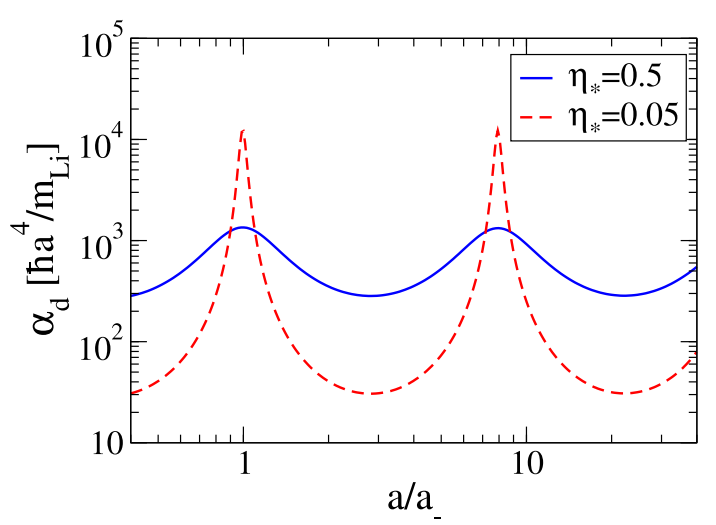

Fig. 8. Universal prediction of $\alpha_{d}$ for the ${ }^{7} \mathrm{Li}^{8}{ }^{87} \mathrm{Rb}$ system at negative interspecies scattering length $a$ in units of $\hbar a^{4} / m_{L i}$ as a function of $a / a_{-}$.

\section{Summary and conclusion}

In this paper, we have calculated the three-body loss rates in heteronuclear mixtures of atoms for the case of large scattering length between the unlike atoms.

We have formulated a universal effective field theory for this system and derived momentum-space integral equations for the trimer energies and the atom-dimer scattering 
$19^{\text {th }}$ International IUPAP Conference on Few-Body Problems in Physics

amplitude. From an analysis of the bound state equation we have calculated the ratio of the resonance positions $a_{*} /\left|a_{-}\right|$ as a function of the mass ratio, $\delta$. Moreover, we have calculated the recombination amplitude numerically and provided semi-analytical expressions for the three-body recombination into shallow and deep dimers as a function of the interspecies scattering length $a$ and the Efimov width parameter $\eta_{*}$. Three-body recombination rates for heteronuclear mixtures were previously calculated by D'Incao and Esry [27]. A comparison with their results will be given in [29].

The expressions in Eqs. $(13,16,22)$ together with the result for $C(\delta)$ in Fig. 5 fully determine the three-body recombination rates for heteronuclear bosonic mixtures with resonant scattering between the unlike atoms in the universal zero-range theory. The atom-dimer relaxation rates are given by Eqs. $(18,20)$. These equations are universal and can be used to analyze experimental data for any combination of atoms with the range applicability of the universal theory.

As an example, we have applied our results to some heteronuclear mixtures in ongoing and planned experiments. For the recent experiment by the Florence group which uses a mixture of ${ }^{41} \mathrm{~K}$ and ${ }^{87} \mathrm{Rb}$ atoms $[28,33]$, we obtain moderate discrepancies between theory and experiment. For the resonance positions, we predict $a_{*} /\left|a_{-}\right|=0.52$ while the experimental ratio is $a_{*} /\left|a_{-}\right|=2.7$. Given that this ratio can lie anywhere between 1 and $\exp \left(\pi / s_{0}\right)=131$, this discrepancy is not too large but no definite conclusion can be drawn at the moment.

Using the value $\eta_{*}=0.12$ extracted in [28], we find that the calculated recombination rate at the resonance is about one order of magnitude too small. Using smaller values of $\eta_{*}$ the size of the experimental rate can be reproduced. In order to resolve this discrepancy, more measurements around the resonance position are required. Currently, there are only two data points and $\eta_{*}$ can not be determined accurately. Additional data would allow for a more precise determination of $\eta_{*}$ and allow for a test of the resonance shape predicted by the universal theory.

Finally, we have calculated the relevant parameters determining the three-body loss rates for various other mixtures and summarized them in Table 1. Extending earlier work by D'Incao and Esry [27,38], our predictions lay the theoretical ground for the experimental observation of Efimov physics in heteronuclear mixtures. They should be useful for planning and analyzing future experiments.

\section{Acknowledgements}

We thank David Canham and Francesco Minardi for discussions. KH was supported by the "Studienstiftung des Deutschen Volkes" and by the Bonn-Cologne Graduate School of Physics and Astronomy. HWH acknowledges support from the BMBF under contract No. 06BN9006.

\section{References}

1. E. Braaten and H.-W. Hammer, Phys. Rept. 428, 259 (2006) [arXiv:cond-mat/0410417].

2. L. Platter, Few Body Syst. 46, 139 (2009) [arXiv:0904.2227 [nucl-th]].

3. V. Efimov, Phys. Lett. 33B, 563 (1970).

4. E. Nielsen and J.H. Macek, Phys. Rev. Lett. 83, 1566 (1999).

5. B.D. Esry, C.H. Greene, and J.P. Burke, Phys. Rev. Lett. 83, 1751 (1999).

6. P.F. Bedaque, E. Braaten, and H.-W. Hammer, Phys. Rev. Lett. 85, 908 (2000) [arXiv:cond-mat/0002365].

7. E. Braaten and H.-W. Hammer, Phys. Rev. Lett. 87, 160407 (2001) [arXiv:cond-mat/0103331].

8. E. Braaten and H.-W. Hammer, Phys. Rev. A 70, 042706 (2004) [arXiv:cond-mat/0303249].

9. T. Kraemer et al., Nature 440, 315 (2006) [arXiv:condmat/0512394].

10. S. Knoop et al., Nature Physics 5, 227 (2009) [arXiv:0807.3306 [cond-mat]].

11. K. Helfrich and H.-W. Hammer, Europhys. Lett. 86, 53003 (2009) [arXiv:0902.3410].

12. H.-W. Hammer and L. Platter, Eur. Phys. J. A 32, 113 (2007) [arXiv:nucl-th/0610105].

13. J. von Stecher, J. P. D'Incao, and C. H. Greene, Nature Physics 5, 417 (2009) [arXiv:0810.3876].

14. F. Ferlaino, S. Knoop, M. Berninger, W. Harm, J. P. D'Incao, H.-C. Nägerl, R. Grimm, Phys. Rev. Lett. 102, 140401 (2009) [arXiv:0903.1276].

15. M. Zaccanti, B. Deissler, C. D’Errico, M. Fattori, M. Jona-Lasinio, S. Müller, G. Roati, M. Inguscio, and G. Modugno, Nature Physics 5, 586 (2009) [arXiv:0810.3876].

16. N. Gross, Z. Shotan, S. Kokkelmans, L. Khaykovich, Phys. Rev. Lett. 103, 163202 (2009) [arXiv:0906.4731].

17. S.E. Pollack, D. Dries, and R.G. Hulet, arXiv:0911.0893 [cond-mat.quant-gas].

18. T. B. Ottenstein, T. Lompe, M. Kohnen, A. N. Wenz, S. Jochim, Phys. Rev. Lett. 101, 203202 (2008) [arXiv:0806.0587 [cond-mat]].

19. J.H. Huckans, J.R. Williams, E.L. Hazlett, R.W. Stites, and K.M. O'Hara, Phys. Rev. Lett. 102, 165302 (2009) [arXiv:0810.3288].

20. E. Braaten, H.-W. Hammer, D. Kang and L. Platter, Phys. Rev. Lett. 103, 073202 (2009) [arXiv:0811.3578].

21. P. Naidon and M. Ueda, Phys. Rev. Lett. 103, 073203 (2009) [arXiv:0811.4086].

22. R. Schmidt, S. Floerchinger, and C. Wetterich, Phys. Rev. A 79, 053633 (2009) [arXiv:0812.1191].

23. A.N. Wenz, T. Lompe, T.B. Ottenstein, F. Serwane, G. Zürn, and S. Jochim, Phys. Rev. A 80, 040702 (2009) [arXiv:0906.4378].

24. J.R. Williams, E.L. Hazlett, J.H. Huckans, R.W. Stites, Y. Zhang, and K.M. O'Hara, arXiv:0908.0789.

25. S. Jochim, private communication.

26. E. Braaten, H. W. Hammer, D. Kang and L. Platter, arXiv:0908.4046 [cond-mat.quant-gas]. 
27. J.P. D'Incao and B.D. Esry, Phys. Rev. A 73, 030702(R) (2006) [arXiv:physics/0508119].

28. G. Barontini, C. Weber, F. Rabatti, J. Catani, G. Thalhammer, M. Inguscio, and F. Minardi, Phys. Rev. Lett. 103, 043201 (2009) [arXiv:0901.4584].

29. K. Helfrich, H.-W. Hammer and D.S. Petrov, in preparation.

30. E. Braaten and H.-W. Hammer, Annals Phys. 322, 120 (2007) [arXiv:cond-mat/0612123].

31. M.A. Efremov, L. Plimak, B. Berg, M.Yu. Ivanov, and W.P. Schleich, Phys. Rev. A 80, 022714 (2009) [arXiv:0905.3974 [quant-ph]].

32. F. Minardi, private communication.

33. G. Barontini et al., Erratum, private communication.

34. C. Marzok, B. Deh, C. Zimmermann, Ph.W. Courteille, E. Tiemann, Y.V. Vanne, and A. Saenz, Phys. Rev. A 79, 012717 (2009) [arXiv:0808.3967 [cond-mat.other]].

35. B. Deh, C. Marzok, C. Zimmermann, and Ph.W. Courteille, Phys. Rev. A 77, 010701(R) (2008) [arXiv:0709.4554 [cond-mat.other]].

36. C. Silber, S. Guenther, C. Marzok, B. Deh, Ph.W. Courteille, and C. Zimmermann, Phys. Rev. Lett. 95, 170408 (2009) [arXiv:cond-mat/0506217].

37. J. Deiglmayr, A. Grochola, M. Repp, K. Mörtlbauer, C. Glück, J. Lange , O. Dulieu, R. Wester, and M. Weidemüller, Phys. Rev. Lett. 101, 133004 (2008) [arXiv:0807.3272 [quant-ph]].

38. J.P. D'Incao and B.D. Esry, Phys. Rev. A 73, 030703(R) (2006) [arXiv:cond-mat/0508474]. 